\title{
Altered PD-L1 Expression in Non-Small Cell Lung Cancer Patient After Induction Chemotherapy: A Case Report
}

\author{
Fadi Nasr ${ }^{\mathrm{a}}$, b, c, e , Ahmad Al Ghoche ${ }^{\mathrm{a}}$, Saada Diaba \\ Souheil Hallitc, Moussa Riachi ${ }^{\mathrm{d}}$
}

\begin{abstract}
Targeting the immune checkpoint of programmed death ligand 1 (PDL1) and programmed cell death protein 1 (PD-1) with immunotherapy has improved the treatment of many tumors including non small cell lung cancer (NSCLC). Checking PD-L1 status must be done during the course of therapy. Changes of PD-L1 status with therapy have been described in several cancer subtypes with different treatment modalities including chemotherapy and immunotherapy. Here we present a case report of a locally advanced squamous lung carcinoma patient presenting a decrease of PD-L1 level after chemotherapy. This case will be added to the few reports describing such changes in PD-L1 expression after different treatment modalities. More data are needed to understand the underlying mechanisms and its treatment implications.
\end{abstract}

Keywords: PD-L1; Chemotherapy; Squamous lung cancer

\section{Introduction}

Lung cancer is the leading cause of cancer-related deaths worldwide [1]. Overall, the chance of developing lung cancer during lifetime is as high as one in 15 males and one in 17 females with a higher incidence in smokers compared to non-smokers. Lebanon has the highest incidence of lung cancer among females and is the third highest among males in the Arab region [2]. Non-small-cell lung cancer (NSCLC) accounts for approximately $80 \%$ of all cases. Concurrent chemo-

Manuscript submitted January 3, 2019, accepted January 18, 2019

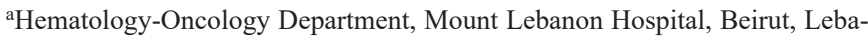
non

bHematology-Oncology Department, Hotel-Dieu de France University Hospital, Faculty of Medicine, Saint Joseph University, Beirut, Lebanon

${ }^{\mathrm{c}}$ Faculty of Medicine and Medical Sciences, Holy Spirit University of Kaslik (USEK), Jounieh, Lebanon

dDepartment of Pulmonary, Hotel-Dieu de France University Hospital, Faculty of Medicine, Saint Joseph University, Beirut, Lebanon

eCorresponding Author: Fadi Nasr, Hematology-Oncology Department, Mount Lebanon Hospital, PO Box 470, Hazmieh, Lebanon.

Email: nasrfadi@hotmail.com

doi: https://doi.org/10.14740/jmc3249 radiation therapy (CCRT) is the treatment of choice for locally advanced NSCLC (LA-NSCLC).

Programmed cell death protein 1 (PD-1) is a protein of the immunoglobulins family, present on the surface of $\mathrm{T}$ cells, B cells and macrophages [3]. It has a downregulating role in the immune response; when expressed by tumor cells, the PD-1 ligands, programmed death ligand 1 (PD-L1) and PD-L2 exert an inhibitory effect on T cells [4]. PD-1 and PD-L1 belong to the immune checkpoint pathway. In tumor environment, PD-L1/PD-1 interaction promotes tumor progression [5]. PD-L1 expression is upregulated in many tumors. PD-1 ligand on the surface of the T-cells interacts with its receptor PD-L1 on the tumor cell, thus inhibiting the anti-tumor activity of the immune system [6]. Nowadays, it is recently found that immune checkpoint inhibitors regulate this mechanism by releasing the block and reactivating the immune system against cancer cells [6-8], thus leading to tumor control by using self-immunity instead of using cytotoxic agents.

With the advances in lung cancer treatment, especially with the addition of checkpoint inhibitors to the therapeutic arsenal thus changing treatment strategies [9] and leading to improved survival in this population [10], checking the PD-L1 status on tumor tissue is being used on regular basis either in the locally advanced or metastatic disease.

\section{Case Report}

Hereby we present a case of a 63-year-old, asymptomatic male patient with coronary artery disease post-angioplasty. A 7-cm necrotic mass was found in March 2018 on injected computed tomography (CT) chest done for suspicious lesion incidentally found on chest X-ray. Bronchoscopy was inconclusive and CT-guided tumor biopsy in April 2018 showed a poorly differentiated squamous lung carcinoma. Positron emission tomography-computed tomography (PET-CT) scan confirmed a Stage IIIC (T3N3M0) disease with left apical mass of $7 \mathrm{~cm}$ with standardized uptake value (SUV) of 25.21 and bilateral hilar and subcarinal adenopathies, the largest measuring $13 \mathrm{~mm}$ with SUV 6.44. Treatment was started and patient was given three cycles of gemcitabine with carboplatin as induction chemotherapy with the aim to render the tumor operable or to continue with definitive radiotherapy according to response. PET-CT scan was done in July 2018 and showed a good response with an irregular spiculated mass of 
$4.3 \mathrm{~cm}$. Hilar adenopathy decreased to $10.3 \mathrm{~mm}$ with SUV dropping to 2.71 with no new findings seen. Following these results, left upper lobectomy with lymph node dissection was done in August 2018. We were left, on the postoperative tissue, with a moderately differentiated keratinizing squamous cell carcinoma with a tumor of $4.5 \mathrm{~cm}$ associated with parietal pleura invasion and presence of vascular emboli. Twenty eight lymph nodes were removed from hilum and subarctic region, anterior mediastinum, and para-esophagus and all were negative for malignancy. PD-L1 before chemotherapy on the initial biopsy was $60 \%$ versus $5 \%$ on the post-operative tissue, using the same Clone Dako 22C3 autostainer for evaluation.

Research involving non-routine analyses was approved by the Institutional Review Board at the Mount Lebanon Hospital and written informed consent obtained from the patient described herein, in accordance with applicable local and international legal and ethical standards, including the Declaration of Helsinki of the World Medical Association.

\section{Discussion}

The case described in this report adds to the increasingly being of change in PD-L1 expression during therapy with either chemotherapy, targeted or immunotherapy [11-14]. Neoadjuvant chemotherapy may up-regulate the expression of PD-L1 in lung squamous cell carcinoma as was recently published [15]. A recently published study showed increased PD-L1 expression on tumor cells in head and neck squamous cell carcinoma treated with TPF induction chemotherapy [16]. Another case report showed a decrease in PD-L1 level in NSCLC after chemotherapy including gemcitabine and platinum [17]. A paper published in Translational Oncology also showed significant induction of PD-L1 expression in esophageal squamous cell carcinoma after standard chemotherapy treatments including paclitaxel, 5-fluorouracil and platinum agents [18]. And even more, in ovarian cancer, it has been reported that chemotherapy induces PD-L1 overexpression via the nuclear factor- $\kappa \mathrm{B}$ [19]. The value of this change in expression may be a prognostic marker that can be used in such cancer cases. Two recent studies have been published and reported an alteration of PD-L1 level (upregulation and downregulation) after using either chemotherapy alone or concomitantly with immunotherapy in locally advanced NSCLC [20], [21]. Therefore, the results are a little bit contradictory regarding the effect of oncologic therapy on PD-L1 expression level. This controversy is to be more investigated in order to define and explain the exact effect of immunotherapy on treated cases according to PD-L1 on tumor cells and to tumor subtype and stage of the disease.

A study evaluating PD-L1 in epidermal growth factor receptor $(E G F R)$ mutant patients showed an increased level of receptors post resistance to gefitinib used in the metastatic setting, thus suggesting the need to rebiopsy after progression on tyrosine kinase inhibitors [22]. The second biopsy is not to only check if T790m mutation is present and probably use osimertinib [23], but also to check the PD-L1 status in order to define the possible benefit from immunotherapy according to expression level. The change in the PD-L1 level with therapy is neither cancer-type dependent nor treatmentdependent.

\section{Conclusions}

In front of available data and the increasing number of cases being reported about such change in PD-L1 level with multiple types of treatment and in different malignancies and stages, we may need to rebiopsy every case on progression and to recheck to PD-L1 expression level and maybe other tumor markers in order to define the best treatment approach especially with the implication of immunotherapy in almost every single step of management regardless of the therapeutic approach used. This change in PD-L1 expression could have an impact on the survival of locally advanced and metastatic cases, either in lung cancer or maybe other solid tumors.

In front of these changes in PD-L1 expression that is being increasingly reported with different treatment modalities and several cancer types, we may need to consider biopsy after failure of each line of therapy and in the transition from neoadjuvant to adjuvant therapy in order to try to define the correct approach for the new line of treatment being considered. Furthermore, since PD-L1 is changing, we may think about retesting for microsatellite instability (MSI) for example on therapy change, among other possible tumor markers that might be interesting. The change in the expression was not limited to chemotherapy or immunotherapy in any tumor stage. And this may lead us to new prognostic scores and will change survival data according to the expression pre and post intervention in locally advanced and metastatic cases not limited to lung cancer. These findings together may change our vision in treating new cancer cases. This needs further investigations and reports.

\section{Acknowledgments}

We would like to thank the patient for his cooperation in this study.

\section{Funding Disclosure}

None.

\section{Conflict of Interest}

The authors have nothing to declare.

\section{Informed Consent}

Written informed consents were obtained from the patient for publication of this case report. 


\section{Author's Contribution}

AG, SD, SH collected data, designed and wrote the manuscript. FN and MR provided relevant scientific advice and revised the manuscript, analyzed and interpreted the patient data regarding clinical presentation, physiopathologic aspects and treatment options. All listed authors contributed to critically revising the article and have approved the final version.

\section{Case Report Guidelines}

This case report conforms with the Consensus-Based Clinical Case Reporting Guideline (http://www.care-statement.org/).

\section{Declarations}

The datasets used and analyzed during the present study are available from the corresponding author on reasonable request.

\section{References}

1. Siegel RL, Miller KD, Jemal A. Cancer statistics, 2016. CA Cancer J Clin. 2016;66(1):7-30.

2. Temraz S, Charafeddine M, Mukherji D, Shamseddine A. Trends in lung cancer incidence in Lebanon by gender and histological type over the period 2005-2008. J Epidemiol Glob Health. 2017;7(3):161-167.

3. Latchman Y, Wood CR, Chernova T, Chaudhary D, Borde M, Chernova I, Iwai Y, et al. PD-L2 is a second ligand for PD-1 and inhibits T cell activation. Nat Immunol. 2001;2(3):261-268.

4. Zhu X, Lang J. Soluble PD-1 and PD-L1: predictive and prognostic significance in cancer. Oncotarget. 2017;8(57):97671-97682.

5. Dong P, Xiong Y, Yue J, Hanley SJB, Watari H. TumorIntrinsic PD-L1 signaling in cancer initiation, development and treatment: beyond immune evasion. Front Oncol. 2018;8:386.

6. Sun C, Mezzadra R, Schumacher TN. Regulation and function of the PD-L1 checkpoint. Immunity. 2018;48(3):434452.

7. Alsaab HO, Sau S, Alzhrani R, Tatiparti K, Bhise K, Kashaw SK, Iyer AK. PD-1 and PD-L1 checkpoint signaling inhibition for cancer immunotherapy: mechanism, combinations, and clinical outcome. Front Pharmacol. 2017;8:561.

8. Jain P, Jain C, Velcheti V. Role of immune-checkpoint inhibitors in lung cancer. Ther Adv Respir Dis. 2018;12:1753465817750075.

9. Bustamante Alvarez JG, Gonzalez-Cao M, Karachaliou N, Santarpia M, Viteri S, Teixido C, Rosell R. Advances in immunotherapy for treatment of lung cancer. Cancer Biol Med. 2015;12(3):209-222.

10. Borghaei H, Langer CJ, Gadgeel S, Papadimitrakopoulou
VA, Patnaik A, Powell SF, Gentzler RD, et al. 24-Month overall survival from KEYNOTE-021 Cohort G: pemetrexed and carboplatin with or without pembrolizumab as first-line therapy for advanced nonsquamous non-small cell lung cancer. J Thorac Oncol. 2019;14(1):124-129.

11. Vilain RE, Menzies AM, Wilmott JS, Kakavand H, Madore J, Guminski A, Liniker E, et al. Dynamic changes in PD-L1 expression and immune infiltrates early during treatment predict response to PD-1 blockade in melanoma. Clin Cancer Res. 2017;23(17):5024-5033.

12. Shin J, Chung JH, Kim SH, Lee KS, Suh KJ, Lee JY, Kim JW, et al. Effect of platinum-based chemotherapy on PD-L1 expression on tumor cells in non-small cell lung cancer. Cancer Res Treat. 2018.

13. Han JJ, Kim DW, Koh J, Keam B, Kim TM, Jeon YK, Lee SH, et al. Change in PD-L1 expression after acquiring resistance to gefitinib in EGFR-mutant non-smallcell lung cancer. Clin Lung Cancer. 2016;17(4):263-270 e262.

14. Omori S, Kenmotsu H, Abe M, Watanabe R, Sugino T, Kobayashi H, Nakashima K, et al. Changes in programmed death ligand 1 expression in non-small cell lung cancer patients who received anticancer treatments. Int J Clin Oncol. 2018;23(6):1052-1059.

15. Song Z, Yu X, Zhang Y. Altered expression of programmed death-ligand 1 after neo-adjuvant chemotherapy in patients with lung squamous cell carcinoma. Lung Cancer. 2016;99:166-171.

16. Leduc C, Adam J, Louvet E, Sourisseau T, Dorvault N, Bernard M, Maingot E, et al. TPF induction chemotherapy increases PD-L1 expression in tumour cells and immune cells in head and neck squamous cell carcinoma. ESMO Open. 2018;3(1):e000257.

17. Sheng J, Fang W, Yu J, Chen N, Zhan J, Ma Y, Yang Y, et al. Expression of programmed death ligand-1 on tumor cells varies pre and post chemotherapy in non-small cell lung cancer. Sci Rep. 2016;6:20090.

18. Ng HY, Li J, Tao L, Lam AK, Chan KW, Ko JMY, Yu VZ, et al. Chemotherapeutic treatments increase PD-L1 expression in esophageal squamous cell carcinoma through EGFR/ERK activation. Transl Oncol. 2018;11(6):13231333.

19. Peng J, Hamanishi J, Matsumura N, Abiko K, Murat $\mathrm{K}$, Baba T, Yamaguchi K, et al. Chemotherapy induces programmed cell death-ligand 1 overexpression via the nuclear factor-kappaB to foster an immunosuppressive tumor microenvironment in ovarian cancer. Cancer Res. 2015;75(23):5034-5045.

20. Fujimoto D, Uehara K, Sato Y, Sakanoue I, Ito M, Teraoka S, Nagata K, et al. Alteration of PD-L1 expression and its prognostic impact after concurrent chemoradiation therapy in non-small cell lung cancer patients. Sci Rep. 2017;7(1):11373.

21. Rojko L, Reiniger L, Teglasi V, Fabian K, Pipek O, Vagvolgyi A, Agocs L, et al. Chemotherapy treatment is associated with altered PD-L1 expression in lung cancer patients. J Cancer Res Clin Oncol. 2018;144(7):12191226.

22. Wu SG, Shih JY. Management of acquired resistance to 
EGFR TKI-targeted therapy in advanced non-small cell lung cancer. Mol Cancer. 2018;17(1):38.

23. Mok TS, Wu YL, Ahn MJ, Garassino MC, Kim HR, Ra- malingam SS, Shepherd FA, et al. Osimertinib or platinum-pemetrexed in EGFR T790M-positive lung cancer. N Engl J Med. 2017;376(7):629-640. 